\title{
Center vs. Periphery
}

By N.N.

\section{- Desideratum}

Evernote keywords: issues tagged for first-priority treatment in this entry

- 6th October

- Alexandria

- Capital vs. Provinces

- countryside, city vs. countryside, rural areas, villages

- empowerment (tamkin)

- geography

- marginal, marginalized groups, marginality and exclusion processess

- metropolis

- Nubian language and culture, Nubian revival, Nubians, first Nubian deputy in Egyptian parliament
- Port Said

- provinces, regions, cultural emancipation of "the South"

- Sfax

- Sohag

- Sousse

- Țantā

- the Șa ${ }^{c} \bar{d}$ (Upper Egypt)

- Upper Beni Sueif

- Upper Minya

- voices from the Delta

Entries pointing here

ARraYs - Downtown/Centre-ville $\diamond$ Dual Identities $/$ Masking $\diamond$ Father Figures $\diamond$ Football $\downarrow$ Garbage $\downarrow$ Gated Communities / Compounds $\downarrow$ Hashish $\downarrow$ Language $\diamond$ Manish msämih $\downarrow$ The Policeman Criminal $\downarrow$ The Voice from Above

CODES - Beautiful vs. Ugly 